\title{
Conditional Reactive Simulatability
}

\author{
Michael Backes $^{1}$, Markus Dürmuth ${ }^{1}$, Dennis Hofheinz ${ }^{2}$, and Ralf Küsters ${ }^{3}$ \\ ${ }^{1}$ Saarland University \\ \{backes, duermuth\}@cs.uni-sb.de \\ ${ }^{2}$ CWI, Cryptology and Information Security Group, Prof. Dr. R. Cramer \\ Dennis.Hofheinz@cwi.nl \\ ${ }^{3}$ Christian-Albrechts-Universität zu Kiel \\ kuesters@ti.informatik.uni-kiel.de
}

\begin{abstract}
Simulatability has established itself as a salient notion for defining and proving the security of cryptographic protocols since it entails strong security and compositionality guarantees, which are achieved by universally quantifying over all environmental behaviors of the analyzed protocol. As a consequence, however, protocols that are secure except for certain environmental behaviors are not simulatable, even if these behaviors are efficiently identifiable and thus can be prevented by the surrounding protocol.

We propose a relaxation of simulatability by conditioning the permitted environmental behaviors, i.e., simulation is only required for environmental behaviors that fulfill explicitly stated constraints. This yields a more fine-grained security definition that is achievable for several protocols for which unconditional simulatability is too strict a notion, or at lower cost for the underlying cryptographic primitives. Although imposing restrictions on the environment destroys unconditional composability in general, we show that the composition of a large class of conditionally simulatable protocols yields protocols that are again simulatable under suitable conditions. This even holds for the case of cyclic assume-guarantee conditions where protocols only guarantee suitable behavior if they themselves are offered certain guarantees. Furthermore, composing several commonly investigated protocol classes with conditionally simulatable subprotocols yields protocols that are again simulatable in the standard, unconditional sense.
\end{abstract}

\section{Introduction}

As a tool to define and prove the security of cryptographic protocols, the concept of simulatability has a long history, e.g., [3423 22[1130]. In recent years, in particular the general simulatability frameworks of reactive simulatability [86] and universal composability [1415] proved useful for analyzing security properties of cryptographic protocols in distributed systems. In such a simulatability framework, a protocol is compared to an ideal specification of the respective protocol task, usually given by a single machine called trusted host that is immune to any adversarial attacks by construction. A protocol is said to be secure, if for every adversary interacting with the real protocol there exists another adversary interacting with the ideal specification such that no protocol environment can distinguish the ideal specification (with the ideal adversary) from the real implementation (with the real adversary). This essentially means that every attack that an adversary may successfully mount against the real implementation

D. Gollmann, J. Meier, and A. Sabelfeld (Eds.): ESORICS 2006, LNCS 4189, pp. 424 443 2006.

(C) Springer-Verlag Berlin Heidelberg 2006 
can be mounted as well against the ideal specification. In that sense, the real protocol is at least as secure as the ideal specification.

This definition is very appealing due to its simplicity, and at the same time it provides very strong security guarantees. Specifically, both mentioned frameworks allow for very general composition theorems (see, e.g., [32 14]7]). In a nutshell, these theorems guarantee that a secure protocol can be composed with arbitrary other protocols and still retains its security. These strong results are essentially entailed by the universal quantification over all protocol environments. However, such strong compositionality properties are bought at the price that several protocol tasks are not securely realizable at all in the sense of simulatability. This includes important cryptographic tasks such as bit commitment, zero-knowledge, oblivious transfer [1614], and (authenticated) Byzantine agreement [29], classes of secure multi-party computation [17], classes of functionalities that fulfill certain game-based definitions [18], and Dolev-Yao style abstractions of symmetric encryption, XOR, and hash functions [3]49].

This nuisance led to several attempts to weaken the simulatability definition, either by strengthening the ideal adversary or by limiting the attack capabilities of the real adversary, which, however, results in restricted adversary models and thus in less realistic scenarios. A more detailed review of related work is given below.

Our Contribution. In this paper, we also endeavor to circumvent a specific class of the aforementioned impossibility results, namely those that arise due to certain environmental behaviors that cannot be properly simulated. The prime example contained in this class is Dolev-Yao style symmetric encryption, i.e., symbolic abstractions of symmetric encryption as constructors of a term algebra with a small set of algebraic properties. This kind of encryption can only be correctly simulated if the protocol using the encryption scheme does not cause a so-called commitment problem. Our approach for circumventing impossibility in these cases does however not follow the prevalent idea of augmenting or constraining the capabilities of the adversary. Instead, we limit the number of protocol environments in which a protocol is required to be secure. This idea applies particularly nicely to protocols that can be securely realized except for certain distinguished environmental behaviors, especially if these behaviors are efficiently identifiable and thus can be prevented by the surrounding protocol; among others, Dolev-Yao style symmetric encryption is of this kind. The resulting security notion is named conditional reactive simulatability. In addition to circumvent known impossibility results for unconditional simulatability, the notion of conditional reactive simulatability may also allow for securely realizing ideal functionalities at lower cost on the underlying cryptographic primitives. For instance, if Dolev-Yao style symmetric encryption permits the construction of key cycles, e.g., encrypting a key with itself, it is only securely realizable by encryption schemes that fulfill certain strong, non-standard assumptions such as dynamic KDM security [5]. When, however, conditioning the functionality to those cases that exclude key cycles, successful simulation based on weaker, more standard security notions such as IND-CCA2 security is possible.

Despite imposing restrictions on the surrounding protocol and thus giving up the universal quantification of environments that allows for general compositionality, we show that the notion of conditional reactive simulatability still entails strong compositionality guarantees. More specifically, we prove that if one composes protocols each 
of which is conditionally simulatable provided that their surrounding protocol fulfills an arbitrary trace property, and if these properties do not give rise to cyclic dependencies, then the composition of these protocols is conditionally simulatable under natural conditions on the (overall) surrounding protocol. Technically, the theorem establishes a cryptographic statement on the acyclic composition of assume-guarantee specifications, i.e., specifications that guarantee suitable behaviors only if they themselves are offered suitable guarantees. Assume-guarantee specifications have been well investigated in the past, mostly for non-security-specific contexts [31,26,1,20] but also specifically for security aspects [24] (but without investigations of simulatability and composition). The postulation of acyclicity applies to most cases in practice, e.g., to protocols that provide specific security guarantees to their subprotocols without making these guarantees dependent on the outputs they obtain from these subprotocols.

Interestingly, we can even prove compositionality for cyclic dependencies of such specifications, i.e., compositions of protocols that mutually promise to adhere to a certain behavior only if they mutually receive guarantees from each other. This case is technically more demanding since an inductive proof by proceeding through the acyclic dependency graph as done in the proof of the acyclic case is no longer possible. In fact, it is easy to show that for cyclic dependencies, subprotocols that are conditionally simulatable under arbitrary trace properties might not be securely composable. However, we prove that the theorem for the acyclic case can be carried over to the cyclic case if the constraints imposed on protocols for conditional simulatability are safety properties. Safety properties arguably constitute the most important class of properties for which conditional simulatability is used, especially since liveness properties usually cannot be achieved unless one additionally constraints the adversary to fair scheduling.

Finally, we note that composing protocol classes with conditionally simulatable subprotocols can yield protocols that are simulatable in the standard, unconditional sense.

Our results are formalized in the Reactive Simulatability framework. However, we do not use any specific characteristics of this framework, so our results can naturally be carried over to the Universal Composability framework.

Related Work. There have been several attempts to relax simulatability to avoid impossibility results. The work closest to ours is the work on proving Dolev-Yao style symmetric encryption sound in the sense of simulatability [3]. There it was shown that Dolev-Yao style symmetric encryption can be securely realized if the environmental protocol does not cause the commitment problem and in addition key cycles are excluded. This definition thus constitutes a special case of conditional reactive simulatability yet without investigating more general conditions or corresponding compositionality aspects. Nevertheless, our work is inspired by their idea of augmenting simulatability with conditions on environments.

The impossibility of simulating a specific bit commitment was shown in [16]. The remedy proposed there was to augment the real protocol with certain "helping trusted hosts" which are, by definition, immune to any attack on the real protocol; thus, effectively this weakens the real adversary. More specifically, [16] presented simulatably secure protocols for bit commitment and zero-knowledge. However, these protocols rely on a so-called Common Reference String (CRS), which is a form of a trusted setup assumption on the protocol participants. In a similar vein, [17] shows that basically 
every trusted host can be realized using a CRS as a helper functionality. One point of criticism against the CRS approach is that the proposed protocols lose security in a formal and also very intuitive sense as soon as the CRS setup assumption is invalidated. The related approach [25] uses a Random Oracle (RO) instead of a CRS to help real protocols achieve simulatable security. The benefit of their construction is that the proposed protocols retain at least classical (i.e., non-simulatable) security properties when the RO assumption is invalidated. However, also there, simulatability in the original sense is lost as soon as this happens.

In [33], the real and ideal adversaries are equipped with a so-called imaginary angel. This is an oracle that (selectively) solves a certain class of hard computational problems for the adversary. Under a very strong computational assumption, this notion could be shown to avoid known impossibility results for simulatability. Yet, as the imaginary angels behave in a very specific way tailored towards precisely circumventing these impossibility results, e.g., these angels make their response dependent on the set of corrupted parties, the model might be considered unintuitive.

In [10], it is shown how to realize any trusted host in a simulatable manner, if the ideal adversary is freed from some of its computational restrictions. However, it is substantial that in their security notion, the ideal adversary is not restricted to polynomial-time, but the real adversary is. So in particular, the security notion they consider is not transitive and it is generally not easy in their framework to construct larger protocols modularly.

Outline. We first review the underlying Reactive Simulatability framework in Section 2 and subsequently define the more fine-grained version of conditional reactive simulatability in Section 3 The bulk of the paper is dedicated to the investigation of the compositionality aspects of this new security notion for both acyclic and cyclic assumeguarantee conditions, which is done in Section 4 The usefulness of conditional reactive simulatability is further exemplified in Section 5 by showing how this notion can be exploited to cryptographically justify common idealizations of cryptography. Section 6 concludes. More details and proofs can be found in our technical report [2].

\section{Review of the Reactive Simulatability Framework}

Our work builds upon the Reactive Simulatability framework. We will briefly review relevant definitions and refer the reader to [8] for details.

\subsection{Overall Framework}

A protocol is modeled as a structure $(M, S)$ consisting of a set of protocol machines and a set of service ports, to which the protocol user connect 1 . Machines are probabilistic, polynomial-time I/O automata, and are connected by ports. The model differentiates inports and out-ports, where each out-port is connected to exactly one in-port by naming convention. Moreover, in- and out-ports may be service or non-service ports. In what

\footnotetext{
${ }^{1}$ Actually, a structure represents a protocol in a specific corruption situation. To handle different corruption situations, systems (i.e., sets of structures) are used. However, in the style of [8]19], we concentrate on a given specific corruption situation for ease of presentation.
} 
follows, by $S^{\text {in }}$ we denote the service in-ports of $S$ and by $S^{C \text {, out }}$ the complement of $M$ 's service out-ports, i.e., the set of service in-ports of machines $M$ connects to.

Two structures $\left(M_{1}, S_{1}\right)$ and $\left(M_{2}, S_{2}\right)$ are composable iff they connect through their respective service ports only. Their composition is given by $\left(M_{1} \cup M_{2}, S\right)$ where $S$ includes all ports from $S_{1}$ and $S_{2}$ that are not connected to another machine in $M_{1} \cup M_{2}$.

A set of machines $M$ is closed iff all ports are connected to corresponding ports of machines that are in the same set. A structure can be complemented to a closed set by a so-called honest user $\mathrm{H}$ and an adversary $\mathrm{A}$, where $\mathrm{H}$ connects to service ports only, and $A$ connects to all remaining open ports, and both machines may interact. The tuple $(M, S, \mathrm{H}, \mathrm{A})$ is then called a configuration of $(M, S)$ where one of the machines $\mathrm{H}$ or A plays the role of the master scheduler, i.e., if no machine was activated by receiving a message, the master schedule is activated. A closed set $C$ is a runnable system. The transcript of a single run is called a trace (often denoted by $\mathbf{t}$ and decorations thereof) and is defined to be a sequence of transitions performed by the machines. A transition of a machine $\mathrm{M}$ is of the form $\left(\bar{p}, s, s^{\prime}, \bar{p}^{\prime}\right)$ where $\bar{p}$ describes the in-ports of $M$ along with the current message written on these ports, $s$ is the current configuration of $M, s^{\prime}$ is a successor configuration (computed depending on $\bar{p}$ and $s$ ), and $\bar{p}^{\prime}$ are the out-ports along with the output produced. We denote by $\operatorname{run}_{C, k}$ the distribution of traces induced by runs of $C$ with security parameter $k$. The restriction $\mathbf{t}\left\lceil_{S}\right.$ of a trace $\mathbf{t}$ to a set of in-ports $S$ is defined in the obvious way. (Note that $\mathbf{t} \Gamma_{S}$ only depends on the first component $(\bar{p})$ of the transitions of $\mathbf{t}$ ). Now, run $_{C, k}\left\lceil_{S}\right.$ denotes the distribution of the traces induced by runs of $C$ with security parameter $k$ when restricted to $S$. The restriction of a trace $t$ to a machine $\mathrm{M}$ is obtained from $\mathbf{t}$ by removing all transitions not done by $\mathrm{M}$. Now, the distribution of such traces given $k$ is denoted by view $w_{C, k}(\mathrm{M})$. We refer to the $k$-indexed family $\left\{\text { view }_{C, k}(\mathrm{M})\right\}_{k}$ of these views by view $w_{C}(\mathrm{M})$.

\subsection{Simulatability}

Simulatability is used in different areas of cryptography. Informally speaking, for reactive systems it says that whatever might happen to a protocol $(M, S)$ can also happen to another protocol $\left(M^{\prime}, S\right)$. Here both protocols need to have the same set of service ports $S$ to allow for a meaningful comparison. Typically, $\left(M^{\prime}, S\right)$ is an idealization, or specification, of the protocol task that $(M, S)$ is to implement. We therefore call $(M, S)$ the real and $\left(M^{\prime}, S\right)$ the ideal protocol. (Typically, the ideal protocol consists only of a single machine $\mathrm{TH}$, a trusted host, that guarantees an ideal behaviour to a user of the protocol.) For simulatability one requires that for every configuration $(M, S, \mathrm{H}, \mathrm{A})$, with honest user $\mathrm{H}$ and real adversary $\mathrm{A}$, there is a configuration $\left(M^{\prime}, S, \mathrm{H}, \mathrm{A}^{\prime}\right)$ of $\left(M^{\prime}, S\right)$, with the same honest user $\mathrm{H}$ and a (possibly different) ideal adversary $A^{\prime}$, such that $H$ cannot distinguish both scenarios. This is illustrated in Figure 1

The notion that $\mathrm{H}$ cannot distinguish both scenarios is captured by the notion of computational indistinguishability: Two families $\left(\operatorname{var}_{k}\right)_{k \in \mathbb{N}},\left(\operatorname{var}_{k}^{\prime}\right)_{k \in \mathbb{N}}$ of random variables on common domains $D_{k}$ are computationally indistinguishable (“ $\approx$ ”) if no polynomialtime algorithm can distinguish both distributions with non-negligible probability, i.e., if for all polynomial-time algorithms Dis the following holds:

$$
\left|\operatorname{Pr}\left[\operatorname{Dis}\left(1^{k}, \operatorname{var}_{k}\right)=1\right]-\operatorname{Pr}\left[\operatorname{Dis}\left(1^{k}, \operatorname{var}_{k}\right)=1\right]\right| \text { is negligible in } k,
$$



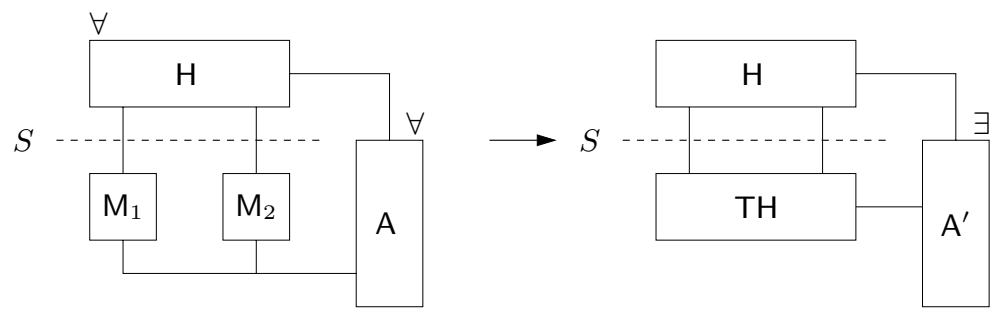

Fig. 1. Simulatability: The two views of $\mathrm{H}$ must be indistinguishable

where a function $g: \mathbb{N} \rightarrow \mathbb{R}_{>0}$ is said to be negligible iff for all positive polynomials $Q, \exists k_{0} \forall k \geq k_{0}: g(k) \leq 1 / Q(k)$.

Definition 1 (Reactive Simulatability). Let structures $(M, S)$ and $\left(M^{\prime}, S\right)$ with identical sets of service ports be given. We write $(M, S) \geq_{\mathrm{sec}}^{\text {poly }}\left(M^{\prime}, S\right)$, where $\geq_{\mathrm{sec}}^{\text {poly }}$ is read as computationally at least as secure as or securely realizes, if for every configuration conf $=(M, S, \mathrm{H}, \mathrm{A})$, there exists a configuration conf ${ }^{\prime}=\left(M^{\prime}, S, \mathrm{H}, \mathrm{A}^{\prime}\right)$ (with the same $\mathrm{H})$ such that

$$
\text { view }_{\text {conf }}(\mathrm{H}) \approx \text { view }_{\text {conf }}(\mathbf{H}) .
$$

One also defines universal simulatability, where $\mathrm{A}^{\prime}$ in conf $f^{\prime}$ does not depend on $\mathrm{H}$, i.e., the order of quantifiers is reversed, and blackbox simulatability, where $\mathrm{A}^{\prime}$ is the composition of a fixed part Sim (the simulator) and A. In the sequel, we omit the superscript poly.

\section{Conditional Reactive Simulatability}

Reactive simulatability (Definition1) permits configurations with arbitrary honest users $\mathrm{H}$ (satisfying some syntactic requirements on ports). In other words, reactive simulatability requires a faithful simulation of the combination of the real adversary and real protocol by the ideal adversary and ideal protocol for every honest user. This universal quantification over all honest users allows for a general composition theorem [32]7], which says that if protocol $(M, S)$ is as secure as protocol $\left(M^{\prime}, S\right)$, then $(M, S)$ can be substituted for $\left(M^{\prime}, S\right)$ in any larger protocol without invalidating simulatability. For this type of compositional property, simulatability can even be shown to be necessary [28].

However, reactive simulatability may be too strict in certain practical scenarios: The simulation might fail for certain honest users, but in the application under consideration such users may not occur since the protocol in question may always be used in a certain (secure) way. For example, consider Dolev-Yao style symmetric encryption. It was shown in [3] that this kind of encryption is not securely realizable in the sense of reactive simulatability, due to the so-called commitment problem: If an encrypted message is sent to the adversary, where the adversary neither knows the message nor the key, the best the simulator can do is to create a new key and encrypt a random message with this key. If later the message becomes known, indistinguishability guarantees that the 
simulation is still correct. However, if later the key becomes known, the simulator has to come up with a suitable key that decrypts the chosen ciphertext to the correct message. This is not possible in general. However, in the application under consideration the way Dolev-Yao style symmetric encryption is used, e.g., by a larger protocol (representing the honest user), may guarantee that the encryption key is never exposed. It turns out that in this situation faithful simulation is still possible.

Following this idea, we propose a relaxation of reactive simulatability, called conditional reactive simulatability, where instead of quantifying over all honest users, we quantify only over those honest users which satisfy a certain condition. In this way troublesome honest users which would not occur in the application anyway can be ruled out.

The conditions on honest users are expressed in terms of what we call predicates. A predicate, which is defined with respect to a set $S$ of ports (typically service in-ports), is a set of sequences of bit strings for every port of $S$. Using predicates, we can restrict the kind and the order of messages on ports of $S$ in a run of a system. To formally define these predicates, we need the following notation: For sets $A$ and $B$, we denote by $B^{A}$ the set of mappings from $A$ to $B$. If $A$ is a finite set, then the elements of $B^{A}$ can be considered to be tuples where every component is an element of $B$ and corresponds to an element of $A$. For $i \geq 0$ and a set $A$, we denote by $A^{i}$ the set of all words over $A$ of length $i$. Now, predicates are defined as follows:

Definition 2 (Predicates). Let $S$ be a set of ports. We call a set $\pi$ with

$$
\pi \subseteq \bigcup_{i \geq 0}\left(\left(\{0,1\}^{*}\right)^{S}\right)^{i} .
$$

a predicate $\pi$ over $S$ if the following conditions are satisfied:

1. If $\pi=s_{1} \cdots s_{i}, s_{j} \in\left(\{0,1\}^{*}\right)^{S}$, then we have that for every $j \in\{1, \ldots, i\}$ there exists $p \in S$ such that $s_{j}(p) \neq \varepsilon$, i.e., for every $s_{j}$ at least one port contains $a$ non-empty message.

2. $\pi$ is decidable in polynomial-time, i.e., there is a probabilistic polynomial-time algorithm that, on input $t$, outputs whether or not $t \in \pi$.

We call $t \in \pi$ an $S$-trace.

Instead of a single predicate, one could also consider a family of predicates indexed by the security parameter. However, for the application presented in this paper, simple predicates suffice. Also, all results presented in this paper easily carry over to the case of families of predicates.

We will use the following notation. We write $\pi=$ true for a predicate $\pi$ over $S$ with $\pi=\bigcup_{i \geq 0}\left(\left(\{0,1\}^{*}\right)^{S}\right)^{i}$. Furthermore, for two predicates $\pi_{1}$ and $\pi_{2}$ over two disjoint port sets $S_{1}$ and $S_{2}$, we write $\pi_{1} \wedge \pi_{2}$ for the predicate containing all $\left(S_{1} \cup S_{2}\right)$-traces such that for every trace in $\pi_{1} \wedge \pi_{2}$ its restriction to $S_{1}$ and $S_{2}$ belongs to $\pi_{1}$ and $\pi_{2}$, respectively 2 Intuitively, $\pi_{1} \wedge \pi_{2}$ represents the conjunction of $\pi_{1}$ and $\pi_{2}$.

An $S$-trace $t^{\prime}$ is a prefix of an $S$-trace $t$ if there exist $t^{\prime \prime}$ such that $t=t^{\prime} \cdot t^{\prime \prime}$ where '.' denotes concatenation. A predicate $\pi$ over $S$ is prefix-closed iff for every $S$-trace $t \in \pi$

${ }^{2}$ In run restricted to some port set $S$, all entries with inputs only in non- $S$ ports are deleted. 
every prefix of $t$ belongs to $\pi$ as well. We also call such a predicate a safety property since once it is violates it stays violated.

Now, we say that a set of machines $M$ fulfills a predicate $\pi$ over a set of ports $S$, if in runs of $M$ with any other set of machines the sequences of messages written on ports in $S$ belong to $\pi$. More precisely, it suffices if this is true with overwhelming probability:

Definition 3 (Predicate Fulfillment). Let $M$ be a set of machines with service ports $S$ and let $\pi$ be a predicate over a subset $S^{\prime}$ of the ports $S^{C, \text { out }}$ of machines to which machines in $M$ connect. Then, $M$ fulfills $\pi$ if for any set of machines $\bar{M}$ such that $C:=\{M, \bar{M}\}$ is closed,

$$
\operatorname{Pr}_{t \leftarrow \operatorname{run}_{C, k}}\left[\left(t\left\lceil_{S^{\prime}}\right) \in \pi\right] \text { is overwhelming as a function in } k .\right.
$$

We are now ready to present the definition of conditional reactive simulatability.

Definition 4 (Conditional Reactive Simulatability). Let structures $(M, S)$ and $\left(M^{\prime}, S\right)$ with identical set $S$ of service ports be given, and let $\pi$ be a predicate over a subset of the service in-ports of $S$. We say that $(M, S)$ is at least as secure as (or realizes) $\left(M^{\prime}, S\right)$ under condition $\pi$ (written $(M, S) \geq_{\mathrm{sec}}^{\pi}\left(M^{\prime}, S\right)$ ) if for every configuration conf $=(M, S, \mathrm{H}, \mathrm{A})$ such that $\mathrm{H}$ fulfills $\pi$, there exists a configuration conf ${ }^{\prime}=\left(M^{\prime}, S, \mathrm{H}, \mathrm{A}^{\prime}\right)$ (with the same $\mathrm{H}$ ) such that

$$
\text { view }_{\text {conf }}(\mathrm{H}) \approx \text { view }_{\text {conf }}(\mathrm{H}) .
$$

Conditional universal simulatability and conditional blackbox simulatability are defined with the obvious modifications.

\section{Composition Under Conditional Reactive Simulatability}

In this section, we present composition theorems for conditional reactive simulatability. As mentioned in the introduction, when composing protocols which assume certain conditions (predicates) to hold on their service in-ports and in turn guarantee certain conditions (predicates) to hold on service in-ports of other protocols, cyclic dependencies may occur. In what follows, we first introduce the general setting (Section 4.2) and then present general composition theorems both for the acyclic and cyclic case (Section 4.2 and 4.3). While for the acyclic case no restrictions on predicates are put, for the cyclic case we require predicates to be safety properties.

\subsection{The General Setting}

One would expect that a protocol $M_{0}$ (for brevity we omit the service ports) that is simulatable under condition $\pi$ can be securely composed with a protocol $M_{1}$ that fulfills $\pi$. In some applications, the larger protocol $M_{1}$ may fulfill $\pi$ only if $M_{1}$ itself is used in a "sane" way, i.e., a predicate, say $\tau$, is fulfilled on the service in-ports of $M_{1}$. Then, one would expect that $M_{0}$ securely composes with $M_{1}$ as long as $\tau$ is fulfilled. More generally, we consider the composition of several protocols with assume-guarantee conditions among them. In what follows, this is formalized.

Let $\pi$ and $\tau$ be predicates over $S_{\pi}$ and $S_{\tau}$, respectively, and let $\mathbf{t}$ be a trace. We say that $\boldsymbol{t}$ satisfies $\tau \rightarrow \pi$ if $\mathbf{t}\left\lceil_{S_{\tau}} \in \tau\right.$ implies $\mathbf{t}\left\lceil S_{\pi} \in \pi\right.$. 
Definition 5 (Conditional Predicate Fulfillment). Let $M$ be a set of machines with service ports $S, \tau$ be a predicate over a subset $S_{\tau}$ of $S^{i n}$, and $\pi$ be a predicate over a subset $S_{\pi}$ of $S^{C, \text { out }}$.

Then, $M$ fulfills $\pi$ under condition $\tau$ if $\tau \rightarrow \pi$ is satisfied with overwhelming probability no matter with which machines $M$ interacts, i.e., for all sets $\bar{M}$ of machines such that $C:=\{M, \bar{M}\}$ is closed, we have that

$$
\operatorname{Pr}_{\boldsymbol{t} \leftarrow \text { run }_{C, k}}[\boldsymbol{t} \text { satisfies } \tau \rightarrow \pi] \text { is overwhelming as a function in } k \text {. }
$$

In what follows, for every $i=1, \ldots, n$, let $P_{i}:=\left(M_{i}, S_{i}\right)$ and $P_{i}^{\prime}:=\left(M_{i}^{\prime}, S_{i}\right)$ be real and ideal protocols, respectively. We consider the following predicates for these protocols.

Let $\tau_{i}^{j}$ be a predicate over $S_{j}^{C, \text { out }} \cap S_{i}^{i n}$ (service in-ports of $P_{i}$ to which $P_{j}$ connects) and $\tau_{i}^{\mathrm{H}}$ be a predicate over $S_{i}^{i n} \backslash \bigcup_{j=1}^{n} S_{j}^{C \text {,out }}$ (service in-ports of $P_{i}$ to which no other protocol connects). Intuitively, $\tau_{i}^{j}$ denotes the guarantees the $i$ th protocol expects from the $j$ th one. Analogously, $\tau_{i}^{\mathrm{H}}$ specifies the guarantees the $i$ th protocol expects from $\mathrm{H}$. (Note that $\mathrm{H}$ may connect to all service in-ports of $P_{i}$ the other protocols do not connect to.) We denote by

$$
\tau_{i}=\tau_{i}^{\mathrm{H}} \wedge \bigwedge_{j \neq i} \tau_{i}^{j}
$$

the guarantees the $i$ th protocol expects from other protocols. Note that $\tau_{i}$ is a predicate over $S_{i}^{\text {in }}$.

Similarly, we now define the guarantees the $i$ th protocol provides to other protocols. Let $\pi_{i}^{j}$ be a predicate over $S_{i}^{C, \text { out }} \cap S_{j}^{i n}$ (service in-ports of $P_{j}$ to which $P_{i}$ connects). Intuitively, $\pi_{i}^{j}$ denotes the guarantees the $i$ th protocol gives to the $j$ th one. Note that we do not consider a predicate $\pi_{i}^{\mathrm{H}}$. This simplifies our presentation and is without loss of generality since we are only interested in the compositionality properties of the composed protocol. We denote by

$$
\pi_{i}=\bigwedge_{j \neq i} \pi_{i}^{j}
$$

the guarantees the $i$ th protocol provides to other protocols. Note that $\pi_{i}$ is a predicate over $\bigcup_{j \neq i}\left(S_{i}^{C, \text { out }} \cap S_{j}^{\text {in }}\right)$.

In order for the composition theorems to hold, we clearly need that

$$
\pi_{j}^{i} \subseteq \tau_{i}^{j}
$$

i.e., the guarantees $\tau_{i}^{j}$ the $i$ th protocol expects from the $j$ th one are actually met by the guarantees $\pi_{j}^{i}$ the $j$ th protocol offers to the $i$ th protocol.

Obviously, in the setting above the guarantees among the protocols may be cyclic: the $i$ th protocol provides guarantee $\pi_{i}^{j}$ (and hence, $\tau_{j}^{i}$ ) to the $j$ th protocol only if the $j$ th protocol guarantees $\tau_{i}^{j}$, and vice versa, i.e., the $j$ th protocol provides guarantee $\pi_{j}^{i}$ (and hence, $\tau_{i}^{j}$ ) to the $i$ th protocol only if the $i$ th protocol guarantees $\tau_{j}^{i}$. Hence, in case $\tau_{j}^{i} \neq$ true and $\tau_{i}^{j} \neq$ true the dependencies between the $i$ th and $j$ th protocol are cyclic. The following is a concrete example. 
Example 1. Say that an encryption system $P_{1}$ guarantees that the secret key is not output in plain as long as this secret key is not submitted as part of a plaintext for encryption. However, a larger protocol $P_{2}$ that uses that encryption system might want to encrypt plaintexts multiple times, possibly tagged with some syntactic type information. In particular, as long as no ciphertext itself contains the secret key in plain, this secret key will not be submitted for encryption. In other words, there is a mutual dependency between $P_{1}$ and $P_{2}$. (Obviously, in this particular case secure composition is possible.)

More generally, cyclic dependencies are defined as follows: Let the (directed) dependency graph $G=(V, E)$ be given by

$$
V=\left\{V_{1}, \ldots, V_{n}\right\}, \quad E=\left\{\left(V_{i}, V_{j}\right): \tau_{i}^{j} \neq \text { true }\right\} .
$$

If $G$ is acyclic, we say that the dependencies between the protocols are acyclic or nonmutual, and otherwise, we say that they are cyclic or mutual.

In the following two subsections, we prove theorems for securely composing protocols, both in the case of acyclic and cyclic dependencies between the protocols. In these theorems we need to argue that the condition $\tau_{i}$ the $i$ th protocol expects to be satisfied are in fact fulfilled when composing all protocols. In case of acyclic dependencies between the protocols, this is possible because the fulfillment of $\tau_{i}$ can be traced back to the conditions satisfied by other protocols or the honest users. In case of cyclic dependencies this is in general not possible because one runs into cycles. However, as we will see, if the predicates involved are safety properties, cyclic dependencies can be resolved. We note that the predicates informally stated in Example 1 are in fact safety predicates.

\subsection{Composition in the Acyclic Case}

In this section, we prove the following general composition theorem for the case of acyclic dependencies between the protocols.

Theorem 1. For every $i=1, \ldots, n$, let $P_{i}=\left(M_{i}, S_{i}\right)$ and $P_{i}^{\prime}=\left(M_{i}^{\prime}, S_{i}\right)$ be protocols as introduced above with $P_{i} \geq_{\text {sec }}^{\tau_{i}} P_{i}^{\prime}$, and assume that $M_{i}^{\prime}$ fulfills $\pi_{i}$ under condition $\tau_{i}$ where $\pi_{i}$ and $\tau_{i}$ are defined as above and condition (3) is satisfied. If the dependencies between the protocols are acyclic, we have, for every $i$, that

$$
P_{1}\|\ldots\| P_{n} \quad \geq_{\text {sec }}^{\tau} \quad P_{1}\|\ldots\| P_{i-1}\left\|P_{i}^{\prime}\right\| P_{i+1}\|\ldots\| P_{n},
$$

where $\tau:=\bigwedge_{j=1}^{n} \tau_{j}^{\mathrm{H}}$. Moreover,

$$
P_{1}\|\ldots\| P_{n} \quad \geq_{\text {sec }}^{\tau} \quad P_{1}^{\prime}\|\ldots\| P_{n}^{\prime} .
$$

Before we prove this theorem, we present useful corollaries of this theorem. The first corollary considers the case of two protocols and it easily follows from Theorem 1 using that $P_{2} \geq_{\text {sec }} P_{2}$. 
Corollary 1 (Conditional Subroutine Composition). Assume that $P_{1} \geq_{\mathrm{sec}}^{\pi} P_{1}^{\prime}$. Let $P_{2}=\left(M_{2}, S_{2}\right)$ be a protocol such that $\left.M_{2} i\right)$ connects to all ports over which $\pi$ is defined and ii) fulfills $\pi$ under condition $\tau$ where $\tau$ is a predicate over the service inports of $P_{2}$ to which $P_{1}$ does not connect. Then,

$$
P_{1}\left\|P_{2} \geq_{\text {sec }}^{\tau} P_{1}^{\prime}\right\| P_{2}
$$

If $\tau=$ true, i.e., $M_{2}$ fulfills $\pi$ unconditionally, we obtain

$$
P_{1}\left\|P_{2} \geq_{\text {sec }} P_{1}^{\prime}\right\| P_{2} \text {. }
$$

Theorem 1 also allows to combine two protocols that are not connected via service ports:

Corollary 2 (Parallel Composition). Assume that $P_{1} \geq_{\text {sec }}^{\pi_{1}} P_{1}^{\prime}$ and $P_{2} \geq_{\text {sec }}^{\pi_{2}} P_{2}$ such that $P_{1}$ and $P_{2}$ are not connected via service ports. Then,

$$
P_{1}\left\|P_{2} \quad \geq_{\mathrm{sec}}^{\pi_{1} \wedge \pi_{2}} \quad P_{1}^{\prime}\right\| P_{2}^{\prime}
$$

Proof of Theorem 1] The proof relies on the following definition:

Definition 6. Let $M, \tau, \pi$ be as in Definition 5. Then, $M$ fulfills $\pi$ under enforced condition $\tau$ if the predicate $\pi$ is true with overwhelming probability when $M$ interacts with machines that fulfill $\tau$, i.e., for all sets $\bar{M}$ of machines that fulfill $\tau$ and such that $C:=\{M, \bar{M}\}$ is closed, it holds that

$$
\operatorname{Pr}_{\boldsymbol{t} \leftarrow \operatorname{run}_{C, k}}[\boldsymbol{t} \text { satisfies } \pi] \text { is overwhelming as a function in } k \text {. }
$$

Obviously, if $M$ fulfills $\pi$ under condition $\tau$, then $M$ fulfills $\pi$ under enforced condition $\tau$.

As a preparation for our proof, note that for $i=1, \ldots, n$, both $M_{i}^{\prime}$ and $M_{i}$ fulfill $\pi_{i}$ under enforced condition $\tau_{i}$. For $M_{i}^{\prime}$, this is clear by assumption, and for $M_{i}$ it follows from $M_{i} \geq_{\mathrm{sec}}^{\tau} M_{i}^{\prime}$. (Assuming that it is not true for $M_{i}$, one obtains an honest user which cannot be simulated, contradicting the assumption that $M_{i} \geq_{\mathrm{sec}}^{\tau} M_{i}^{\prime}$.) Now fix $i \in\{1, \ldots, n\}$ and set

$$
\tilde{P}_{i}:=P_{1}\|\ldots\| P_{n} \text { and } \tilde{P}_{i}^{\prime}:=P_{1}\|\ldots\| P_{i-1}\left\|P_{i}^{\prime}\right\| P_{i+1}\|\ldots\| P_{n} .
$$

Theorem statement (5): We need to show that for every configuration conf $=\left(\tilde{P}_{i}, \mathrm{H}, \mathrm{A}\right)$ of $\tilde{P}_{i}$, where $\mathrm{H}$ fulfills $\tau$, there is a valid configuration conf $f^{\prime}=\left(\tilde{P}_{i}^{\prime}, \mathrm{H}, \mathrm{A}^{\prime}\right)$ of $\tilde{P}_{i}^{\prime}$ with the same $\mathrm{H}$ such that

$$
\operatorname{view}_{\operatorname{conf}}(\mathrm{H}) \approx \operatorname{view}_{\text {conf }}(\mathrm{H}) \text {. }
$$

Step 1: We construct a new user $\mathrm{H}_{i}$ as a combination of $\mathrm{H}$ with all protocol machines $M_{j}$ except for $M_{i}$. Note that $\mathrm{H}_{i}$ is polynomial-time, so in any case, conf ${ }_{i}:=\left(P_{i}, \mathrm{H}_{i}, \mathrm{~A}\right)$ is a configuration of $P_{i}$. 
$\mathrm{H}_{i}$ fulfills $\tau_{i}$ : Note that this statement makes sense because $\mathrm{H}_{i}$ connects to all of $M_{i}$ 's service ports. Due to space limitations, the somewhat technical proof is only presented in the technical report [2]. In this proof we use that $M_{i}$ fulfills $\pi_{i}$ under enforced condition $\tau_{i}$.

Step 2: Now, since $\mathrm{H}_{i}$ fulfills $\tau_{i}$, the conditional simulatability of $M_{i}$ guarantees the existence of a configuration $\operatorname{conf}_{i}^{\prime}:=\left(P_{i}^{\prime}, \mathrm{H}_{i}, \mathrm{~A}^{\prime}\right)$ with

$$
\text { view }_{\text {conf }_{i}}\left(\mathrm{H}_{i}\right) \approx \text { view }_{\text {conf }_{i}^{\prime}}\left(\mathrm{H}_{i}\right) .
$$

In particular, this yields

$$
\text { view }_{\operatorname{conf}_{i}}(\mathrm{H}) \approx \text { view }_{\text {conf }_{i}^{\prime}}(\mathrm{H})
$$

for the submachine $\mathrm{H}$ of $\mathrm{H}_{i}$.

Step 3: Decomposing $\mathrm{H}_{i}$ into $\mathrm{H}$ and the machines $M_{j}(j \neq i)$ yields a valid configuration $\left(\tilde{P}_{i}^{\prime}, \mathrm{H}, \mathrm{A}^{\prime}\right)$ of protocol $\tilde{P}_{i}^{\prime}$ such that (7) follows from (8) as desired.

Theorem statement (6): We show

$$
P_{1}^{\prime}\|\ldots\| P_{i-1}^{\prime}\left\|P_{i} \ldots\right\| P_{n} \quad \geq_{\text {sec }}^{\tau} \quad P_{1}^{\prime}\|\ldots\| P_{i}^{\prime}\left\|P_{i+1} \ldots\right\| P_{n}
$$

for $i=1, \ldots, n$ by repeatedly applying (5). The case $i=1$ is directly implied by (5), and for $i>1$, all $P_{j}$ with $j<i$ can be set to $P_{j}^{\prime}$. Then by transitivity, (9) implies (6), which completes the proof.

\subsection{Dealing with Mutual Dependencies - Composition in the Cyclic Case}

In this section, we show that protocols can securely be composed even in case of cyclic dependencies given that the predicates considered are safety properties.

Theorem 2. For every $i=1, \ldots, n$, let $P_{i}=\left(M_{i}, S_{i}\right)$ and $P_{i}^{\prime}=\left(M_{i}^{\prime}, S_{i}\right)$ be protocols as introduced in Section 4.1 with $P_{i} \geq_{\mathrm{sec}}^{\tau_{i}} P_{i}^{\prime}$, and assume that $M_{i}^{\prime}$ and $M_{i}$ fulfills $\pi_{i}$ under condition $\tau_{i}$ where $\pi_{i}$ and $\tau_{i}$ are defined as in Section 4.1 and condition (3) is satisfied. Also, assume that all predicates $\tau_{i}^{j}, \tau_{i}^{H}$, and $\pi_{i}^{j}$ are safety properties. Then, for all $i$, we have:

$$
P_{1}\|\ldots\| P_{n} \quad \geq_{\text {sec }}^{\tau} \quad P_{1}\|\ldots\| P_{i-1}\left\|P_{i}^{\prime}\right\| P_{i+1}\|\ldots\| P_{n},
$$

where $\tau:=\bigwedge_{j=1}^{n} \tau_{j}^{\mathrm{H}}$. Moreover,

$$
P_{1}\|\ldots\| P_{n} \quad \geq_{\text {sec }}^{\tau} \quad P_{1}^{\prime}\|\ldots\| P_{n}^{\prime}
$$

We note that in Theorem 2 the requirement that $M_{i}$ fulfills $\pi_{i}$ under condition $\tau_{i}$ can be dispensed with if service out-ports are scheduled locally (which in most scenarios is the case): The reason is that, as in the proof of Theorem 1 it easily follows that if $M_{i}^{\prime}$ fulfills $\pi_{i}$ under condition $\tau_{i}$, then $M_{i}$ fulfills $\pi_{i}$ under enforced condition $\tau_{i}$. Now, it is not hard to see that if service out-ports are scheduled locally, then the notion of Definition 6implies the one of Definition 5. Hence, $M_{i}$ fulfills $\pi_{i}$ under condition $\tau_{i}$. 
Proof of Theorem 2] For the proof of Theorem 2, we need some terminology. For a trace $\mathbf{t}$ and predicates $\tau$ and $\pi$ such that $\tau$ and $\pi$ are safety properties, we say that $t$ satisfies $\tau \rightarrow \pi$ at any time if $\mathbf{t}^{\prime}$ satisfies $\tau \rightarrow \pi$ for every prefix $\mathbf{t}^{\prime}$ of $\mathbf{t}$.

Definition 7. Let $M, \pi, \tau$ be as in Definition 5 such that $\pi$ and $\tau$ are safety properties. Then, $M$ fulfills $\pi$ under condition $\tau$ at any time if the predicate $\tau \rightarrow \pi$ is satisfied at any time with overwhelming probability, no matter with which machines $M$ interacts, i.e., for all sets $\bar{M}$ such that $C:=\{M, \bar{M}\}$ is closed, it holds that

$$
\operatorname{Pr}_{t \leftarrow \text { run }_{C, k}}[\boldsymbol{t} \text { satisfies } \tau \rightarrow \pi \text { at any time }] \text { is overwhelming as a function in } k \text {. }
$$

We can show that the above notion is equivalent to the one defined in Definition 5 .

Lemma 1. Let $M, \pi$, and $\tau$ be as in Definition 7 and such that $M$ contains no master scheduler. Then we have that $M$ fulfills $\pi$ under condition $\tau$ at any time iff $M$ fulfills $\pi$ under condition $\tau$.

Proof. The implication from left to right is obvious. To see the converse direction, let $\bar{M}$ be a set of machines such that $C=\{M, \bar{M}\}$ is closed and let the polynomial $p(k)$ bound the runtime of $\bar{M}$. (Note that $\bar{M}$ necessarily contains a master scheduler.) First, by definition, if a trace $\mathbf{t}$ of $C$ does not satisfy $\tau \rightarrow \pi$ at any time, then there exists a prefix $\mathbf{t}^{\prime}$ of $\mathbf{t}$ which does not satisfy $\tau \rightarrow \pi$, i.e., $\mathbf{t}^{\prime} \Gamma_{S_{\tau}} \in \tau$ but $\mathbf{t}^{\prime}\left\lceil S_{\pi} \notin \pi\right.$. Let $\mathbf{t}^{\prime}$ be of minimal length with this property. It is easy to see that the last transition of $\mathbf{t}^{\prime}$ must be a transition of $\bar{M}$. Now, assume that (12) is not satisfied, i.e., $\operatorname{Pr}_{\mathbf{t} \leftarrow \operatorname{run}_{C, k}}[\mathbf{t}$ does not satisfy $\tau \rightarrow \pi$ at any time] is a non-negligible function in $k$. Consider the machine $\bar{M}^{*}$ which simulates $\bar{M}$ but at the beginning randomly chooses a position $i \in\{1, \ldots, p(k)+1\}$ and when activated for the $i$ th time it stops (simulating $\bar{M}$ ). Let $C^{*}=\left\{M, \bar{M}^{*}\right\}$. Intuitively, $\bar{M}^{*}$ has a high probability to stop a run of $C^{*}$ exactly when the trace produced so far does not satisfy $\tau \rightarrow \pi$. In fact, using that 12 is not satisfied it is easy to verify that $\operatorname{Pr}_{\mathbf{t} \leftarrow \text { run }_{C^{*}, k}}[\mathbf{t}$ does not satisfy $\tau \rightarrow \pi]$ is a non-negligible function in $k$. This implies that $M$ does not fulfill $\pi$ under condition $\tau$.

We can now prove Theorem 2 . For an overview of the proof, see Figure 2 . We first prove (10), from which then (11) follows as in the proof of Theorem 1 Fix $i \in\{1, \ldots, n\}$ and set

$$
\tilde{P}_{i}:=P_{1}\|\ldots\| P_{n} \text { and } \tilde{P}_{i}^{\prime}:=P_{1}\|\ldots\| P_{i-1}\left\|P_{i}^{\prime}\right\| P_{i+1}\|\ldots\| P_{n} .
$$

We need to show that for every configuration conf $=\left(\tilde{P}_{i}, \mathrm{H}, \mathrm{A}\right)$ of $\tilde{P}_{i}$, where $\mathrm{H}$ fulfills $\tau$, there is a valid configuration conf $f^{\prime}=\left(\tilde{P}_{i}^{\prime}, \mathrm{H}, \mathrm{A}^{\prime}\right)$ of $\tilde{P}_{i}^{\prime}$ with the same $\mathrm{H}$, such that

$$
\text { view }_{\text {conf }}(\mathrm{H}) \approx \text { view }_{\text {conf }}(\mathrm{H}) \text {. }
$$

Step 1: We construct a new user $\mathrm{H}_{i}$ as a combination of $\mathrm{H}$ with all protocol machines $M_{j}$ except for $M_{i}$. Note that $\mathrm{H}_{i}$ is polynomial-time, so in any case, conf ${ }_{i}:=\left(P_{i}, \mathrm{H}_{i}, \mathrm{~A}\right)$ is a configuration of $P_{i}$. 

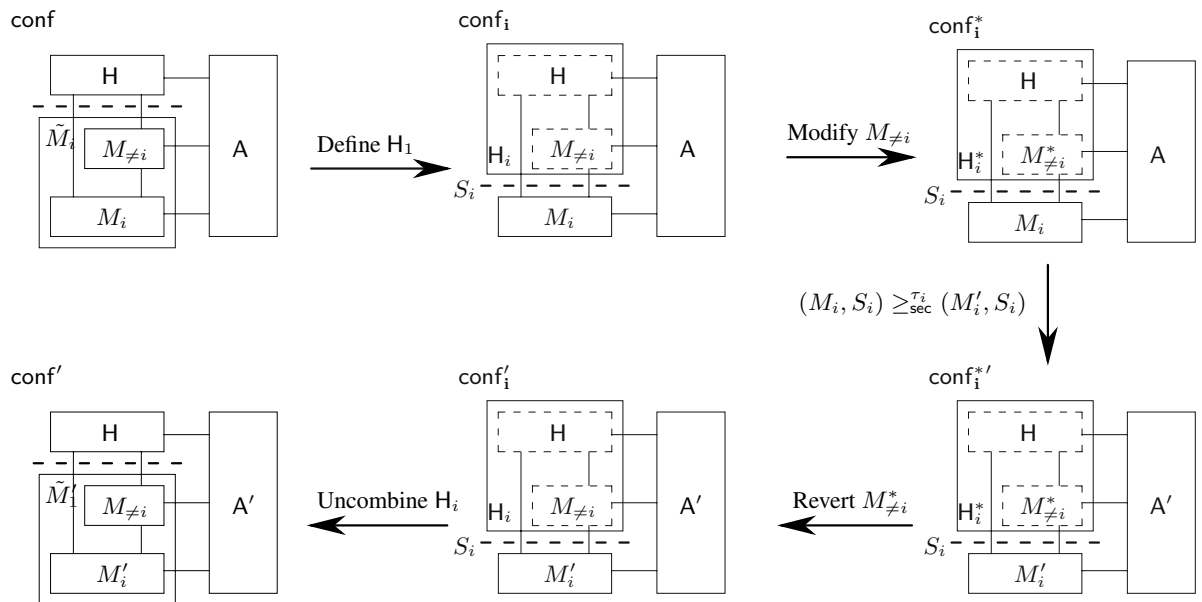

Fig. 2. Overview of the proof of Theorem 2

Step 2: We modify $\mathrm{H}_{i}$ into a new user $\mathrm{H}_{i}^{*}$ such that $\mathrm{H}_{i}^{*}$ fulfills $\tau_{i}$. This is done by substituting all sets of submachines $M_{j}(j \neq i)$ of $\mathrm{H}_{i}$ by sets of machines $M_{j}^{*}$ that fulfill their respective predicates $\pi_{j}$ without any preconditions. More specifically, $M_{j}^{*}$ simulates $M_{j}$ and in addition checks whether $\tau_{j}$ is fulfilled, i.e., whether the observed sequence of inputs on in-ports of $M_{j}$ lies in $\tau_{j}$. By assumption, this can be done efficiently. If $\tau_{j}$ is not fulfilled, then $M_{j}^{*}$ halts immediately.

First claim regarding $\mathrm{H}_{i}^{*}$ : We claim that the view of the submachine $\mathrm{H}$ of $\mathrm{H}_{i}$ is not changed (non-negligibly) by this modification, i.e., we claim

$$
\operatorname{view}_{\operatorname{conf}_{i}}(\mathbf{H}) \approx \operatorname{view}_{\operatorname{conf}_{i}^{*}}(\mathbf{H})
$$

where $\operatorname{conf}_{i}^{*}=\left(P_{i}, \mathrm{H}_{i}^{*}, \mathrm{~A}\right)$.

Assume for contradiction that (14) does not hold. Then the probability that some $\tau_{j}$ $(j \neq i)$ is not fulfilled in a run of $\operatorname{conf}_{i}$ is non-negligible (since otherwise, $\operatorname{conf}_{i}$ and conf $_{i}^{*}$ behave identical). Let $j$ be such that $\tau_{j}$ is with non-negligible probability the first of all predicates $\tau_{\ell}(1 \leq \ell \leq n)$ to become false in a run of conf ${ }_{i}$. By "first", we mean that there is a prefix of the considered run that does not lie in $\tau_{j}$, but all shorter prefixes lie in all $\tau_{\ell}$. (Note that by the prefix-closeness of all $\tau_{\ell}$ such a prefix must exist for some j.)

Because of (1), there is thus a $\tau_{j}^{r}$ (with $r \in\{1, \ldots, n, \mathrm{H}\} \backslash\{j\}$ ) such that with non-negligible probability, $\tau_{j}^{r}$ becomes false before any other predicate $\tau_{\ell}, \ell \neq j$, and $\tau_{j}^{r^{\prime}}, r^{\prime} \neq r$, does. As $r=\mathrm{H}$ directly contradicts the assumption on $\mathrm{H}$, we may assume $r \neq \mathrm{H}$.

Now by assumption, $M_{r}$ fulfills $\pi_{r}$, and thus, by (3) and (1), also $\tau_{j}^{r}$ under condition $\tau_{r}$ (in the sense of Definition 5). By Lemma 1 and the just derived statement about $\tau_{j}^{r}$, this implies that with non-negligible probability, $\tau_{r}$ is false before $\tau_{j}$ is. This is a contradiction to the choice of $j$. 
Second claim regarding $\mathrm{H}_{i}^{*}$ : We claim that $\mathrm{H}_{i}^{*}$ fulfills $\tau_{i}$ (without any precondition). By (1) and the assumption on $\mathrm{H}$, it suffices to prove that for any $j \neq i, M_{j}^{*}$ fulfills $\tau_{i}^{j}$ without any precondition. Now since $M_{j}$ fulfills $\pi_{j}$ under condition $\tau_{j}$, it also does so at any time (Lemma 1). That is, it holds with overwhelming probability that at any point during a run of $M_{j}, \pi_{j}$ is true unless $\tau_{j}$ becomes false.

By construction, $M_{j}^{*}$ and $M_{j}$ behave identically unless $\tau_{j}$ becomes false. That is, also $M_{j}^{*}$ fulfills $\pi_{j}$ under condition $\tau_{j}$ at any time. In particular, by definition of $M_{j}^{*}$, with overwhelming probability $\pi_{j}$ is true when $M_{j}^{*}$ halts. It is also easy to see that $\pi_{j}$ cannot become false after $M_{j}^{*}$ has halted. Hence, $M_{j}^{*}$ fulfills $\pi_{j}$, and thus, $\tau_{i}^{j}$ unconditionally.

Step 3: As $\mathrm{H}_{i}^{*}$ fulfills $\tau_{i}$, the conditional simulatability of $M_{i}$ guarantees the existence of a configuration $\operatorname{conf} f_{i}^{* \prime}:=\left(P_{i}^{\prime}, \mathrm{H}_{i}^{*}, \mathrm{~A}^{\prime}\right)$ with

$$
\operatorname{view}_{\operatorname{conf}}^{*}\left(\mathrm{H}_{i}^{*}\right) \approx \operatorname{view}_{\operatorname{conf}_{i}^{* \prime}}\left(\mathrm{H}_{i}^{*}\right) .
$$

In particular, this yields

$$
\operatorname{view}_{\operatorname{conf}_{i}^{*}}(\mathrm{H}) \approx \operatorname{view}_{\operatorname{conf}_{i}^{* \prime}}(\mathrm{H})
$$

for the submachine $\mathrm{H}$ of $\mathrm{H}_{i}^{*}$.

Step 4: We substitute $\mathrm{H}_{i}^{*}$ again by $\mathrm{H}_{i}$. Since, by assumption, $M_{i}^{\prime}$ fulfills $\pi_{i}$ under condition $\tau_{i}$, analogously to Step 2 we can show that

$$
\text { view }_{\operatorname{conf}_{i}^{* \prime}}(\mathrm{H}) \approx \text { view }_{\operatorname{conf}_{i}^{\prime}}(\mathrm{H})
$$

where $\operatorname{conf}_{i}^{\prime}=\left(P_{i}^{\prime}, \mathrm{H}_{i}, \mathrm{~A}^{\prime}\right)$.

Step 5: Decomposing $\mathrm{H}_{i}$ into $\mathrm{H}$ and the machines $M_{j}(j \neq i)$ yields a valid configuration $\left(\tilde{P}_{i}^{\prime}, \mathrm{H}, \mathrm{A}^{\prime}\right)$ of protocol $\tilde{P}_{i}^{\prime}$ such that (13) and thus (10) follows from (14), 15) and (16) as desired.

\section{Applications and Examples}

In this section, we provide examples substantiating the claim that conditional reactive simulatability constitutes a suitable security notion for circumventing known impossibility results of simulating interesting abstractions of cryptography. In addition, we illustrate that imposing suitable constraints on the environment may allow for a simulation proof based on much weaker assumptions on the underlying cryptography. Generally speaking, conditional reactive simulatability allows for exploiting knowledge of which protocol class will use the protocol under investigation, resulting in more finegrained reasoning about cryptographic protocols.

More specifically, we prove that Dolev-Yao style abstractions of symmetric encryption can be correctly simulated by conditioning environments to those cases that do not cause a so-called commitment problem. For unconditional simulatability, Dolev-Yao style symmetric encryption is known not to be simulatable at all [3]. If one further constraints the environment not to create key cycles, e.g., encrypting a key with itself, we can even establish conditional simulatability based on considerably weaker assumptions 
on the underlying cryptographic encryption scheme. Finally, we show that conditional simulatability may naturally entail unconditional simulatability for composed protocols again.

\subsection{Conditional Simulatability of Dolev-Yao Style Symmetric Encryption}

For Dolev-Yao style symmetric encryption, the following so-called commitment problem inherently prevents the successful application of unconditional reactive simulatability. The ideal encryption system must somehow allow that secret keys are sent from one participant to another. This is used for example in key-exchange protocols. If the ideal system simply allows keys to be sent at any time (and typical Dolev-Yao models do allow all valid terms to be sent at any time), the following problem can occur: An honest participant first sends a ciphertext such that the adversary can see it, and later sends both the contained plaintext and the key. This behavior may even be reasonably designed into protocols, e.g., the ciphertext might be an encrypted bet that is later opened. The simulator will first learn in some abstract way that a ciphertext was sent and has to simulate it by some bitstring, which the adversary sees. Later the simulator sees abstractly that a key becomes known and that the ciphertext contains a specific application message. It cannot change the application message, thus it must simulate a key that decrypts the old ciphertext bitstring (produced without knowledge of the application message) to this specific message.

We omit a rigorous definition of the absence of the commitment problem for DolevYao style symmetric encryption as given in [35] but only give an informal definition for the sake of readability:

\section{Definition 8 (No Commitment Property of Dolev-Yao Style Symmetric Encryp-} tion, informally). The No Commitment property NoComm of Dolev-Yao style symmetric encryption consists of those traces of Dolev-Yao style symmetric encryption that satisfy the following trace predicate: If a term is encrypted at time $t_{1}$ in this trace by an honest user $u$ with secret key sk, and at this time sk is not known to the adversary, then the adversary does not learn the key sk at any future time $t_{2}$ in this trace.

Technically, the requirement that an adversary does not learn certain keys relies on the state of the Dolev-Yao model which keeps track of who knows which term; thus Definition 8 is syntactically not a predicate in the sense of Definition 2 . However, those parts of the state that capture if an adversary already knows keys generated by honest users are uniquely determined by the preceding inputs at the service in-ports. Thus NoComm can naturally be recast as a property that is only defined at the service in-ports of the Dolev-Yao model and thus as a predicate in the sense of Definition 2 (however with a much more tedious notation).

The main result of [5] provides a simulation for those cases in which NoComm is fulfilled provided that the cryptographic encryption scheme fulfills the notion of dynamic KDM security [5]. We can now rephrase their result in our formalism to benefit from the compositionality guarantees entailed by our composition theorems. In the following, let $\left(\left\{\mathrm{TH}_{\mathcal{H}}^{\text {cry_sym,id }}\right\}, S_{\mathcal{H}}\right)$ and $\left(\left\{\mathrm{M}_{\mathcal{E}, u}^{\text {cry_sym,real }} \mid u \in \mathcal{H}\right\}, S_{\mathcal{H}}\right)$ denote the Dolev-Yao model of symmetric encryption and its cryptographic realization from [3]5], respectively, for a set $\mathcal{H} \subseteq\{1, \ldots, n\}$ of honest users, and an encryption scheme $\mathcal{E}$. 
Theorem 3 (Conditional Reactive Simulatability of Dolev-Yao Style Symmetric Encryption). For all symmetric encryption schemes $\mathcal{E}$ that satisfy dynamic KDM security, and for all sets $\mathcal{H} \subseteq\{1, \ldots, n\}$ of honest users, the realization of the Dolev-Yao model is at least as secure as the Dolev-Yao model under condition NoComm, i.e., $\left(\left\{\mathrm{M}_{\mathcal{E}, u}^{\text {cry_sym,real }} \mid u \in \mathcal{H}\right\}, S_{\mathcal{H}}\right) \geq_{\text {sec }}^{\text {NoComm }}\left(\left\{\mathrm{TH}_{\mathcal{H}}^{\text {cry_sym, id }}\right\}, S_{\mathcal{H}}\right)$.

\subsection{Securely Realizing Dolev-Yao Style Symmetric Encryption with Weaker Cryptography}

While Theorem 3 shows that Dolev-Yao style symmetric encryption can be conditionally simulated by excluding the commitment property, it still relies on the strong assumption that the underlying encryption scheme satisfies dynamic KDM security a very strong, non-standard notion for which no realization in the standard model of cryptography is known. However, it turns out that this strong notion is only necessary to deal with the quite exotic case that symmetric keys are encrypted in a cyclic manner, e.g., a key with itself. Most protocols however avoid such constructions by definition, and indeed further constraining simulatability to traces that do not contain key cycles yields a simulatability result based on considerably weaker assumptions on the underlying encryption scheme. More precisely, it suffices that the encryption scheme satisfies indistinguishability under adaptive chosen-ciphertext attacks as well as integrity of ciphertexts. This is the standard security definition of authenticated symmetric encryption [13 12], and efficient symmetric encryptions schemes provably secure in this sense exist under reasonable assumptions [2127].

Definition 9 (No Key Cycles for Dolev-Yao Style Symmetric Encryption, informally). The No Key Cycles property NoKeyCycles of Dolev-Yao style symmetric encryption consists of those traces of Dolev-Yao style symmetric encryption in which honest users do not create encryptions $E\left(s k_{i}, m_{i}\right)$ such that $s k_{i+1}$ is a subterm of $m_{i}$ for $i=0, \ldots, j-1$ for some $j$, and $s k_{0}$ is a subterm of $m_{j}$.

Theorem 4 (Conditional Reactive Simulatability of Dolev-Yao Style Symmetric Encryption w/o Key Cycles). For all authenticated symmetric encryption schemes $\mathcal{E}$ and all sets $\mathcal{H} \subseteq\{1, \ldots, n\}$ of honest users, the realization of the Dolev-Yao model is at least as secure as the Dolev-Yao model under condition NoComm $\wedge$ NoKeyCycles, i.e., $\left(\left\{\mathrm{M}_{\mathcal{E}, u}^{\text {cry_sym,real }} \mid u \in \mathcal{H}\right\}, S_{\mathcal{H}}\right) \geq_{\text {sec }}^{\text {NoComm } \wedge \text { NoKeyCycles }}\left(\left\{\mathrm{TH}_{\mathcal{H}}^{\text {cry_sym,id }}\right\}, S_{\mathcal{H}}\right)$.

\subsection{Simulatable Protocols from Conditionally Simulatable Subprotocols}

We finally illustrate, exploiting Corollary 1 that conditional simulatability can often be turned into unconditional simulatability again (and in fact, it seems hard to come up with a non-artificial example for which Corollary 1 does not apply). Consider a secure channel between two parties that uses Dolev-Yao style symmetric encryption as a subprimitive, which itself is only conditionally simulatable. The secure channel consists of two machines $\mathrm{M}_{1}$ and $\mathrm{M}_{2} . \mathrm{M}_{1}$ expects a message $m$ as input at a service port in?, and encrypts this message with a symmetric key $k$ shared with $\mathrm{M}_{2}$. The encryption is computed using Dolev-Yao style symmetric encryption as a subprimitive, i.e., $m$ 
is output at a service port enc_out ${ }_{1}$ ! and the resulting encryption $e$ is obtained at a service port enc_in ${ }_{1}$ ?. $M_{2}$ outputs the message at a service port out!. We do not give a rigorous definition of this behavior here since this would presuppose introducing a significant amount of notion from [3] but it should be clear already that this secure channel neither causes a commitment problem nor any key cycles by construction. Let $\left(M^{\mathrm{sc}}, S^{\mathrm{sc}}\right):=\left(\left\{\mathrm{M}_{1}, \mathrm{M}_{2}\right\},\left\{\right.\right.$ in? out!, enc_out 1 !, enc_in $\left.\left.{ }_{1} ?\right\}\right)$ denote the secure channel.

Theorem 5. For all authenticated symmetric encryption schemes $\mathcal{E}$, and for $\mathcal{H}=\{1,2\}$, the secure channel based on the realization is unconditionally at least as secure as the secure channel based on the Dolev-Yao model, i.e., $\left(M^{\text {sc }}, S^{\text {sc }}\right)\left\|\left(\left\{\mathrm{M}_{\mathcal{E}, u}^{\text {cry_sym,real }} \mid u \in \mathcal{H}\right\}, S_{\mathcal{H}}\right) \geq_{\text {sec }}\left(M^{\text {sc }}, S^{\text {sc }}\right)\right\|\left(\left\{\mathrm{TH}_{\mathcal{H}}^{\text {cry_sym,id }}\right\}, S_{\mathcal{H}}\right)$.

\section{Conclusion}

We presented a relaxation of simulatability, one of the central concepts of modern cryptography for defining and analyzing the security of multi-party protocols, by permitting to constrain environments to adhere to certain behaviors. The resulting notion is called conditional reactive simulatability. It constitutes a more fine-grained security notion that is achievable i) for protocols for which traditional simulatability is too strong a notion, and ii) based on weaker requirements on the underlying cryptography. In addition, conditional reactive simulatability maintains the interesting property that for various protocol classes, composition of conditionally simulatable protocols yield protocols that are simulatable in the traditional sense.

We furthermore showed that despite imposing restrictions on the surrounding protocol and thus giving up the universal quantification of environments that naturally allowed for compositionality proofs in earlier works, the notion of conditional reactive simulatability still entails strong compositionality guarantees. In particular, this holds for the common case of composing so-called assume-guarantee specifications, i.e., specifications that are known to behave properly if offered suitable inputs, provided that these assumptions and guarantees constitute arbitrary trace properties that do not give rise to cyclic dependencies. We further investigated the theoretically more demanding (but arguably practically less interesting) case of cyclic dependencies among such specifications and proved a similar composition theorem under the additional assumption that conditions are expressible as safety properties.

Acknowledgments. We thank Martín Abadi for interesting discussions.

\section{References}

1. Martín Abadi and Leslie Lamport. Conjoining specifications. ACM Transactional on Programmming Languages and Systems., 17(3):507-534, 1995.

2. M. Backes, M. Dürmuth, D. Hofheinz, and R. Küsters. Conditional Reactive Simulatability. Technical Report 132, Cryptology ePrint Archive, 2006. Online available at http: / / eprint.iacr.org/2006/132.ps. 
3. Michael Backes and Birgit Pfitzmann. Symmetric encryption in a simulatable dolev-yao style cryptographic library. In 17th IEEE Computer Security Foundations Workshop, Proceedings of CSFW 2004, pages 204-218. IEEE Computer Society, 2004.

4. Michael Backes and Birgit Pfitzmann. Limits of the cryptographic realization of Dolev-Yaostyle XOR. In Sabrina De Capitani di Vimercati, Paul F. Syverson, and Dieter Gollmann, editors, Computer Security, Proceedings of ESORICS 2005, number 3679 in Lecture Notes in Computer Science, pages 178-196. Springer-Verlag, 2005.

5. Michael Backes, Birgit Pfitzmann, and Andre Scedrov. Key-dependent message security under active attacks. ePrint Archive, 2005/421, 2006.

6. Michael Backes, Birgit Pfitzmann, and Michael Waidner. A composable cryptographic library with nested operations. In 10th ACM Conference on Computer and Communications Security, Proceedings of CCS 2003, pages 220-230. ACM Press, 2003. Extended abstract.

7. Michael Backes, Birgit Pfitzmann, and Michael Waidner. A general composition theorem for secure reactive systems. In Moni Naor, editor, Theory of Cryptography, Proceedings of TCC 2004, number 2951 in Lecture Notes in Computer Science, pages 336-354. Springer-Verlag, 2004.

8. Michael Backes, Birgit Pfitzmann, and Michael Waidner. Secure asynchronous reactive systems. IACR ePrint Archive, March 2004.

9. Michael Backes, Birgit Pfitzmann, and Michael Waidner. Limits of the Reactive Simulatability/UC of Dolev-Yao models with hashes. Cryptology ePrint Archive 2006/068, 2006.

10. Boaz Barak and Amit Sahai. How to play almost any mental game over the net - concurrent composition via super-polynomial simulation. In 46th Annual Symposium on Foundations of Computer Science, Proceedings of FOCS 2005, pages 543-552. IEEE Computer Society, 2005.

11. Donald Beaver. Foundations of secure interactive computing. In Joan Feigenbaum, editor, Advances in Cryptology, Proceedings of CRYPTO '91, number 576 in Lecture Notes in Computer Science, pages 377-391. Springer-Verlag, 1992.

12. Mihir Bellare and Chanathip Namprempre. Authenticated encryption: Relations among notions and analysis of the generic composition paradigm. In Advances in Cryptology - ASIACRYPT 2000, pages 531-545, 2000.

13. Mihir Bellare and Phillip Rogaway. Encode-then-encipher encryption: How to exploit nonces or redundancy in plaintexts for efficient constructions. In Advances in Cryptology - ASIACRYPT 2000, pages 317-330, 2000.

14. Ran Canetti. Universally composable security: A new paradigm for cryptographic protocols. In 42th Annual Symposium on Foundations of Computer Science, Proceedings of FOCS 2001, pages 136-145. IEEE Computer Society, 2001.

15. Ran Canetti. Universally composable security: A new paradigm for cryptographic protocols. IACR ePrint Archive, January 2005. Full and revised version of [14].

16. Ran Canetti and Marc Fischlin. Universally composable commitments. In Joe Kilian, editor, Advances in Cryptology, Proceedings of CRYPTO 2001, number 2139 in Lecture Notes in Computer Science, pages 19-40. Springer-Verlag, 2001.

17. Ran Canetti, Yehuda Lindell, Rafail Ostrovsky, and Amit Sahai. Universally composable two-party and multi-party secure computation. In 34th Annual ACM Symposium on Theory of Computing, Proceedings of STOC 2002, pages 494-503. ACM Press, 2002. Extended abstract.

18. Anupam Datta, Ante Derek, John C. Mitchell, Ajith Ramanathan, and Andre Scedrov. Games and the impossibility of realizable ideal functionality. In To appear in Proceedings of Theory of Cryptography (TCC 2006), 2006.

19. Anupam Datta, Ralf Küsters, John C. Mitchell, and Ajith Ramanathan. On the relationships between notions of simulation-based security. In In Theory of Cryptography, Proceedings of TCC 2005, pages 476-494, 2005. 
20. Dimitra Giannakopoulou, Corina S. Pasareanu, and Jamieson M. Cobleigh. Assumeguarantee verification of source code with design-level assumptions. In Proceedings 26th International Conference on Software Engineering, pages 211-220, 2004.

21. Virgil D. Gligor and Pompiliu Donescu. Fast encryption and authentication: Xcbc encryption and xecb authentication modes. In Proceedings 8th Fast Software Encryption", pages 82$108,2001$.

22. Oded Goldreich, Silvio Micali, and Avi Wigderson. How to play any mental game-a completeness theorem for protocols with honest majority. In Nineteenth Annual ACM Symposium on Theory of Computing, Proceedings of STOC 1987, pages 218-229. ACM Press, 1987. Extended abstract.

23. Shafi Goldwasser, Silvio Micali, and Charles Rackoff. The knowledge complexity of interactive proof systems. SIAM Journal on Computing, 18(1):186-208, 1989.

24. Heather Hinton. Composing partially-specified systems. In IEEE Symposium on Security and Privacy, Proceedings of SSP 1998, pages 27-39. IEEE Computer Society, 1998.

25. Dennis Hofheinz and Jörn Müller-Quade. Universally composable commitments using random oracles. In Moni Naor, editor, Theory of Cryptography, Proceedings of TCC 2004, number 2951 in Lecture Notes in Computer Science, pages 58-76. Springer-Verlag, 2004.

26. C. Jones. Specification and design of (parallel) programs. In Information Processing 83: Proceedings 9th IFIP World Congress, pages 321-322, 1983.

27. Charanjit Jutla. Encryption modes with almost free message integrity. In Advances in Crptology - EUROCRYPT 2001, pages 529-544, 2001.

28. Yehuda Lindell. General composition and universal composability in secure multi-party computation. In 44th Annual Symposium on Foundations of Computer Science, Proceedings of FOCS 2003, pages 394-403. IEEE Computer Society, 2003.

29. Yehuda Lindell, Anna Lysyanskaya, and Tal Rabin. On the composition of authenticated byzantine agreement. In 34th Annual ACM Symposium on Theory of Computing, Proceedings of STOC 2002, pages 514-523. ACM Press, 2002.

30. Silvio Micali and Phillip Rogaway. Secure computation. In Joan Feigenbaum, editor, Advances in Cryptology, Proceedings of CRYPTO '91, number 576 in Lecture Notes in Computer Science, pages 392-404. Springer-Verlag, 1992. Abstract.

31. J. Misra and M. Chandy. Proofs of networks of processes. IEEE Transactions of Software Engineering, 7(4):417-426, 1981.

32. Birgit Pfitzmann and Michael Waidner. A model for asynchronous reactive systems and its application to secure message transmission. In IEEE Symposium on Security and Privacy, Proceedings of SSP 2001, pages 184-200. IEEE Computer Society, 2001.

33. Manoj Prabhakaran and Amit Sahai. New notions of security: Achieving universal composability without trusted setup. In 36th Annual ACM Symposium on Theory of Computing, Proceedings of STOC 2004, pages 242-251. ACM Press, 2004.

34. Andrew Chi-Chih Yao. Theory and applications of trapdoor functions. In 23th Annual Symposium on Foundations of Computer Science, Proceedings of FOCS 1982, pages 80-91. IEEE Computer Society, 1982. 